\title{
Programas de continuidad de cuidados: el ejemplo del distrito de Chamartín de Madrid
}

\author{
Ana González Rodríguez \\ Centro de Salud Mental de Chamartín, Madrid
}

\begin{abstract}
Resumen
Se presenta el programa de continuidad de cuidados del Centro de Salud Mental de Chamartín en Madrid, para ilustrar con una experiencia fuera del ámbito anglosajón donde surgen estos programas, la teoría expuesta en la primera parte acerca del surgimiento, desarrollo y filosofía de los programas de continuidad de cuidados. Se describen los recursos sanitarios, sociales y personales de los que dispone la población a la que va dirigido y la forma de intervención, con el tipo de actuaciones que se realizan. Por último, se señalan algunos riesgos en el desarrollo y mantenimiento de los programas de continuidad de cuidados.
\end{abstract}

Palabras claves: tratamiento asertivo comunitario; continuidad de cuidados; atención comunitaria en salud mental.

\begin{abstract}
Continuity of care programs: the example of Madrid Chamartin district. The case management program of Chamartin Mental Health Center in Madrid is presented to illustrate with an experience outside the Anglo-Saxon context, where such programs arise, the theory advanced in the first part about the emergence, development and philosophy of continuity of care programs. Finally, some risks in the development and maintenance of continuity of care programs are pointed out. The health, social and staff resources are described, and also the population to be targeted, the form of intervention and the type of actions that are performed. Finally, we point out some risks in the development and maintenance of continuity of care programs.

Keywords: assertive community treatment; case management; community-oriented mental health care.
\end{abstract}

$\mathrm{E}$ n la primera parte de este trabajo, "Programas de continuidad de cuidados: éxitos, fracasos y retos futuros", se revisan las bases teóricas y se repasa la bibliografía existente sobre los programas de continuidad de cuidados, modelo que se ha convertido en piedra angular del tratamiento comunitario de la enfermedad mental grave. Los programas de continuidad de cuidados y sus versiones de tratamiento asertivo comunitario surgieron en EEUU, de ahí se diseminaron a los países anglosajones y posteriormente al resto del mundo. A España llegan a finales de los años ochenta, extendiéndose de manera desigual y con características diferenciales por las distintas Comunidades Autónomas, impulsados por los procesos de reforma psiquiátrica.

El objetivo de este trabajo es describir el funcionamiento de uno de esos programas. El que se desarrolla en un distrito de la ciudad de Madrid.

Chamartín es un distrito de la ciudad de Madrid, con una población aproximada de 140.000 habitantes, 120.000 adultos y 20.000 menores de 18 años, de clase media preferentemente acomodada. El sistema de atención sanitaria, como el de todo el estado español, es público y universal y el modelo de salud mental un "modelo de atención balanceada" según la definición recogida por Thornicroft et al. (2010). Es decir, un modelo que integra elementos comunitarios y hospitalarios, con una mayoría de servicios prestados en la comunidad, próximos a la población. La base sobre la que se sustenta la atención en Salud Mental dentro del distrito es el Centro de Salud Mental (CSM), lugar donde se encuentran los profesionales responsables de cada paciente y eje de la derivación y coordinación con los distintos recursos.

A continuación se describen los distintos elementos del programa de continuidad de cuidados de este distrito de Madrid y su forma de funcionamiento: Recursos sanitarios, sociales y comunitarios del distrito; Equipo de continuidad de cuidados/ Equipo de apoyo social y comunitario; Población diana; Plan de intervención: protocolo de continuidad de cuidados; e, Actuaciones

\section{Recursos sanitarios, sociales y comunitarios del} distrito

Los recursos con los que cuenta el distrito se enumeran a continuación, para dar una idea de la complejidad de la red, 


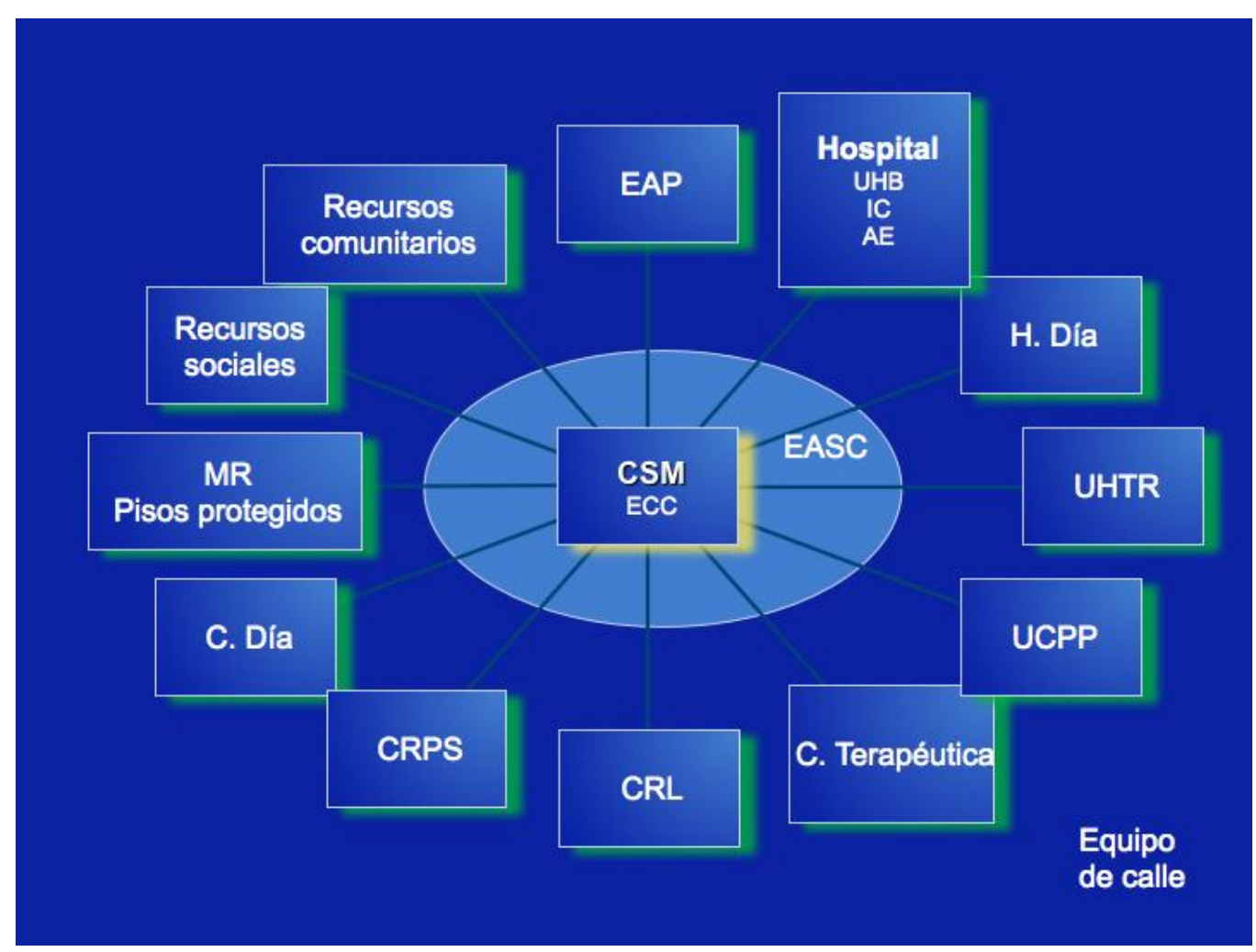

Figura 1. Red de Atención a la Salud Mental del distrito de Chamartín

aunque no se detallan sus características ni funcionamiento, porque escapa del objetivo de este trabajo.

\section{Equipo de continuidad de cuidados/Equipo de apoyo social y comunitario (EASC)}

El equipo de continuidad de cuidados está integrado por una parte del personal del CSM, dependiente de la Consejería de Sanidad, que llamaremos a partir de aquí equipo de continuidad de cuidados original (ECC) y por el equipo de apoyo social comunitario (EASC), de incorporación más reciente, dependiente administrativamente de la Consejería de Familia y Asuntos Sociales y cuyo trabajo se sitúa fuera del centro. El ECC lo forma personal del CSM: tres enfermeros (uno a tiempo parcial), un trabajador social, tres terapeutas ocupacionales (con una dedicación al programa del 10\% de su jornada) y un psiquiatra (dedica un $30 \%$ de su actividad) que actúa como coordinador del equipo. Los enfermeros, trabajador social y terapeutas ocupacionales desempeñan labores de "coordinadores de cuidados" de los pacientes incluidos en el programa. El documento elaborado por la Oficina Regional de Coordinación de Salud Mental especifica las funciones del coordinador de cuidados: "coordina a los distintos profesionales y recursos; mantiene la continuidad de la atención al paciente; actúa como provisor directo de cuidados y vela por la calidad de vida y los derechos de los pacientes" (Consejería de Sanidad, 2002, p.78)

El criterio de adjudicación de coordinador de cuidados a cada paciente se hace en función de las características del paciente y de la disponibilidad del profesional. Un criterio importante a tener en cuenta a la hora de seleccionar el coordinador es la empatía que se establece entre paciente y coordinador. Algunos estudios sugieren que la alianza entre paciente y coordinador de cuidados puede influir en una respuesta favorable. Hay estudios sobre las preferencias de los pacientes y satisfacción con el tratamiento, señalando que los pacientes valoran más la relación con el coordinador de cuidados que los aspectos estructurales del programa comunitario (McGrew, Wilson, \& Bond, 1996; Solomon \& Draine, 1994). Muchos estudios relacionan el grado de consideración de la alianza con la evolución (Gehrs \& Goering, 1994; Klinkenberg, Calsyn, \& Morse, 1998; Neale \& Rosenheck, 1995).

Los equipos de apoyo social y comunitario (EASC) se ponen en marcha en Madrid como parte del Plan de Atención Social a personas con enfermedad mental grave y crónica (20032007), desarrollado por la Consejería de Familia y Asuntos Sociales (Consejería de Servicios Sociales, 2003). Estos equipos compuestos por 4 profesionales están pensados para dar apoyo a 30 pacientes, complementando en el entorno comunitario el trabajo de los equipos de continuidad de cuidados de los CSM. Sus objetivos son: apoyar el mantenimiento del usuario en el propio domicilio y en el entorno socio-comunitario y familiar; promover el desarrollo de capacidades de autonomía personal, social y manejo comunitario; evitar la marginación y el abandono; apoyar la utilización de recursos; mejorar la convivencia familiar y aliviar situaciones de sobrecarga de las familias; colaborar en el acompañamiento y vinculación a la red de salud mental y servicios sociales.

En nuestro centro, desde el momento que se crea el EASC de zona, se consideró que tenía que estar integrado y trabajar 
Tabla 1

Recursos área 2

\begin{tabular}{lll}
\hline Recursos sanitarios & Recursos sociales & Recursos comunitarios \\
\hline $\begin{array}{l}\text { Centro de Salud Mental, eje de la } \\
\text { atención comunitaria. }\end{array}$ & $\begin{array}{l}\text { Centro de Servicios. Sociales del } \\
\text { distrito }\end{array}$ & Educativos \\
Unidad de Hospitalización Breve & $\begin{array}{l}\text { Centro rehabilitación psicosocial, } \\
\text { con } 20 \text { plazas }\end{array}$ & Culturales \\
Hospital de Día & Centro de Día & Formativo-laborales \\
$\begin{array}{l}\text { Unidad Hospitalaria de Tratamiento } \\
\text { Rehabilitador }\end{array}$ & $\begin{array}{l}\text { Centro rehabilitación laboral, } \\
\text { con 10 plazas para el distrito }\end{array}$ & De ocio \\
Equipo de atención primaria & Mini-Residencia abierta, con 12 & \\
plazas residenciales & Pisos protegidos & \\
Atención especializada & &
\end{tabular}

Hospital General de Referencia ${ }^{\mathrm{d}}$

Comunidad Terapéutica $^{\mathrm{e}}$

Unidad de cuidados psiquiátricos

prolongados $^{\mathrm{g}}$

Recursos agencia antidroga

Equipo de calle ${ }^{\mathrm{h}}$

Piso supervisado ${ }^{\mathrm{i}}$

Nota. ${ }^{a}$ Unidad de Hospitalización Breve: situada en el hospital general de referencia para el distrito, con 12 camas.

${ }^{\mathrm{b}}$ Unidad Hospitalaria de Tratamiento Rehabilitador: para pacientes con escasa conciencia de enfermedad y adherencia al tratamiento, que rechazan el tratamiento de rehabilitación psicosocial ambulatorio o éste ha fracasado.

${ }^{c}$ Equipo de atención primaria: donde se encuentran los médicos de atención primaria.

${ }^{\mathrm{d}}$ Hospital general de referencia: en el que además de la unidad de hospitalización psiquiátrica, está la interconsulta (IC) psiquiátrica y otros especialistas médicos (AE, atención especializada).

${ }^{\text {e}}$ Comunidad terapéutica para trastornos de personalidad, con 1 plaza para el distrito.

${ }^{\mathrm{f}}$ Centro de día: para pacientes con mayor nivel de funcionamiento y mayor grado de deterioro psicosocial, con 15 plazas para el distrito.

${ }^{\mathrm{g}}$ Unidad de cuidados psiquiátricos prolongados: Centro psiquiátrico residencial, para pacientes con gran deterioro y necesidad de cuidados

psiquiátricos en un medio residencial de mayor contención y estancia prolongada y/o indefinida.

${ }^{\text {h}}$ Equipo de calle: equipo de continuidad de cuidados independiente, específico para enfermos mentales sin hogar, que tiene el objetivo de integrarlos en la red de salud mental normalizada. Depende de la Consejería de Sanidad y cubre toda la población enferma mental sin hogar de Madrid.

${ }^{i}$ Pisos supervisados: piso para enfermos mentales, con supervisión sanitaria del equipo de Salud Mental.

${ }^{j}$ Pisos protegidos: piso para enfermos mentales con supervisión de servicios sociales.

conjuntamente con el ECC, que ya funcionaba en el centro desde años antes, como única forma de hacer una verdadera continuidad de cuidados, sin solapar actividades y sin dejar vacíos por cubrir. Por ello, aunque dependientes de administraciones distintas, se considera un equipo con actividades diferentes, con una coordinación fluida y reuniones periódicas de puesta en común de los casos. El EASC sólo se ocupa de una parte de los pacientes incluidos en continuidad de cuidados (30 usuarios), que por sus características requieren una intervención intensiva en el medio comunitario._Si hubiera que establecer el tipo de modelo de continuidad de cuidados que se desarrolla en el CSM de Chamartín, podríamos decir que la intervención con la mayoría de los pacientes se ajusta al modelo clínico, salvo en una minoría de pacientes, en que el abordaje es más intensivo y requieren de EASC. En estos últimos el tipo de intervención se corresponde con un tratamiento asertivo comunitario.
No está establecida una ratio de personal: pacientes, aunque en la literatura oscila entre 1:50 en los modelos de tipo agencia de servicios a 1:10 en los modelos asertivos comunitarios. Es obvio que no todos los pacientes requieren la misma intensidad de intervención, ni ésta es igual a lo largo de toda la evolución del proceso. Una buena manera de valorar la necesidad ratio personal: pacientes, es la propuesta que se hizo en el plan de continuidad de cuidados del área 9 de la Comunidad de Madrid, donde la intensidad de la intervención y, por tanto, las ratios de personal más altas, se valoran en función de estado clínico del paciente, el grado de disfunción familiar y el grado de desvinculación del centro (Sobrino, Moreno, \& Desviat, 2007).

\section{Población diana}

El plan de Salud Mental de la Comunidad de Madrid se 2003-2008, mantenido en el de 2010-2014 (Consejería de 
Tabla 2

Criterios de periodicidade para el seguimiento

\begin{tabular}{|c|c|c|c|}
\hline Situacion & \multicolumn{3}{|c|}{ Nivel de Prioridad } \\
\hline $\begin{array}{l}\text { A1. Alto riesgo } \\
\text { con disfunción familiar grave }\end{array}$ & Prioridad 1 & Prioridad 1 & Prioridad 2 \\
\hline $\begin{array}{l}\text { A2. Alto riesgo } \\
\text { sin apoyo (vive solo) }\end{array}$ & Prioridad $1^{\text {a }}$ & Prioridad $1^{\mathrm{a}}$ & Prioridad 2 \\
\hline $\begin{array}{l}\text { A3. Alto riesgo, } \\
\text { convivencia familiar normal }\end{array}$ & Prioridad $2^{\mathrm{a}}$ & Prioridad $3^{\mathrm{b}}$ & Prioridad $4^{\mathrm{b}}$ \\
\hline $\begin{array}{l}\text { A4. Riesgo, } \\
\text { con disfunción familiar grave }\end{array}$ & Prioridad $2^{\mathrm{a}}$ & Prioridad $3^{\mathrm{b}}$ & Prioridad $4^{\mathrm{b}}$ \\
\hline $\begin{array}{l}\text { A5.Riesgo, } \\
\text { sin apoyo (vive solo) }\end{array}$ & Prioridad $2^{\mathrm{a}}$ & Prioridad $3^{\mathrm{b}}$ & Prioridad 4 \\
\hline $\begin{array}{l}\text { A6. Riesgo, } \\
\text { convivencia familiar normal }\end{array}$ & Prioridad $4^{\mathrm{b}}$ & Prioridad $5^{\mathrm{b}}$ & Prioridad $5^{\mathrm{b}}$ \\
\hline $\begin{array}{l}\text { A7.Estable } \\
\text { con disfunción familiar grave }\end{array}$ & Prioridad $5^{\mathrm{b}}$ & Prioridad $6^{\mathrm{c}}$ & Prioridad $6^{\mathrm{c}}$ \\
\hline $\begin{array}{l}\text { A8. Estable } \\
\text { sin apoyo (vive solo) }\end{array}$ & Prioridad $7^{\mathrm{c}}$ & Prioridad $7^{\mathrm{c}}$ & Prioridad $8^{c}$ \\
\hline \multirow[t]{2}{*}{$\begin{array}{l}\text { A9. Estable, } \\
\text { convivencia familiar normal }\end{array}$} & Prioridad $8^{\mathrm{c}}$ & Prioridad $8^{\mathrm{c}}$ & Prioridad $8^{\mathrm{c}}$ \\
\hline & $\begin{array}{c}\text { B3 Desvinculado de los } \\
\text { servicios }\end{array}$ & $\begin{array}{l}\text { B2 Vinculación } \\
\text { ambivalente }\end{array}$ & B1 Buena vinculación \\
\hline
\end{tabular}

Notas.

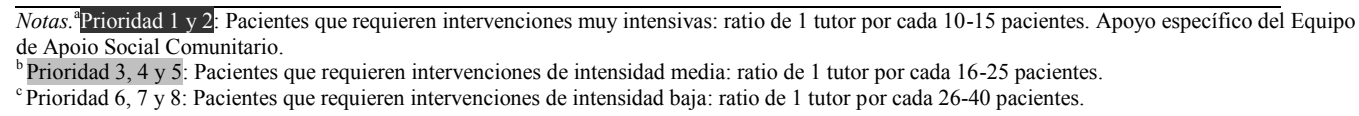

Sanidad, 2010) establece como población diana "los pacientes adultos que están siendo atendidos en los Servicios de Salud Mental de Distrito y que están incluidos en el Programa de Rehabilitación"; "Que presentan deterioro, déficit o minusvalías causadas por su enfermedad mental o en riesgo de padecerlos" y "que requieren o se prevé que van a requerir el uso simultáneo o sucesivo de diferentes recursos y dispositivos sea de la red sanitaria o social u otros de su entorno". Esto incluye los distintos tipos de Psicosis (CIE 9 295, 296, 297 y 298) y “otros diagnósticos psiquiátricos (no derivados de enfermedad médica o neurológica ni discapacidad intelectual) que presenten un alto índice de deterioro psico-social". Es decir, se trata de personas con enfermedad mental grave y persistente (EMGP), fundamentalmente psicosis esquizofrénica, que a consecuencia de la misma presentan un deterioro en sus capacidades y en su nivel de funcionamiento psicosocial, lo que les lleva a tener dificultades para su desenvolvimiento autónomo en la comunidad y por tanto para su integración social. En el distrito de Chamartín hay 201 pacientes que cumplen las características anteriores y están incluidos en continuidad de cuidados; 30 de ellos necesitan una intervención más intensiva y tienen seguimiento por parte

\section{del EASC.}

\section{Plan de intervención: protocolo de continuidad de cuidados}

Una vez que se decide que un paciente es candidato a entrar en el programa de continuidad de cuidados, hay que elaborar un plan específico e individualizado de intervención, acordado entre el coordinador de cuidados, el paciente y el responsable clínico. Este plan de intervención debe quedar reflejado en el protocolo de continuidad de cuidados, de forma que pueda ser revisado y evaluado periódicamente. La Oficina Regional de Coordinación de Salud Mental, estableció un protocolo de continuidad de cuidados, elaborado por un grupo de expertos, con el objetivo de que se implantara en todo Madrid y existiera una cierta homogeneidad entre unas áreas y otras. Este protocolo cuenta con: Ficha carpeta con: datos sociodemográficos; datos clínicos; área familiar; evaluación de necesidades; áreas de intervención; Cuestionario de evaluación de discapacidades y necesidades; y Hojas del plan individualizado de actuaciones con objetivos, personas que las desarrollaran y plazo de intervención y evaluación. 


\section{Actuaciones}

Para conseguir los objetivos acordados, en este programa se realizan quatro tipos de actuaciones:

Actividades centradas en el paciente. Evaluación y diseño del Plan de Intervención; Acompañamiento, asesoramiento y apoyo; control de adherencia al tratamiento; psicoeducación; grupos de apoyo; visitas a domicilio.

Actividades centradas en el entorno. asesoramiento, apoyo y psicoeducación a la familia; asesoramiento y apoyo a otras personas relacionadas con el paciente; antenimiento y desarrollo de soportes comunitarios individualizados; asesoría y defensa del paciente; promoción del asociacionismo; contacto con el sistema judicial y seguimiento de incapacidades y tutelas.

Actividades centradas simultáneamente en el caso y en el entorno. intervención en crisis; evaluación y control de resultados.

Actividades de coordinación y seguimiento con dispositivos sanitarios y sociales. con atención primaria; atención especializada de salud; con el Hospital de Día; Unidades de Hospitalización Breve; Recursos Específicos de Rehabilitación; recursos específicos de rehabilitación dependientes de la Consejería de Familia y Asuntos Sociales; recursos comunitarios (educativos, culturales, formativo-laborales, de ocio, etc.); coordinación Interna; Reuniones del equipo con los responsables clínicos para la derivación de casos al programa y el seguimiento de los mismos; reuniones con el resto del equipo del CSM; gestión de las ayudas económicas para los pacientes. (Programa de Continuidad de Cuidados y Apoyo Comunitario de los Servicios de Salud Mental del Área 9. Leganés, 2007).

\section{Consideraciones Finales}

La enfermedad mental representa una carga importante para las personas que la sufren y sus familiares, tanto en lo referente a calidad como a esperanza de vida. Los servicios de salud mental de calidad son fundamentales para reducir esa carga. Aunque parece existir bastante consenso internacional en cuanto a que los programas de continuidad de cuidados desarrollados a lo largo de los últimos treinta años, son los que mejor garantizan la calidad de cuidados para los enfermos mentales graves y persistentes, siguen existiendo riesgos en su implantación, desarrollo y mantenimiento. La necesidad de coordinar políticas, planes y programas que den servicios completos y de calidad al conjunto de la población, resulta difícil en momentos de crisis y reducción del gasto, cuando la privatización se convierte en la panacea del ahorro y de la rentabilidad.
Como ya se ha señalado, sin el apoyo político no es posible el desarrollo de estos programas, pero tampoco sin la implicación de los profesionales. En un primer momento, el desarrollo de los programas de continuidad de cuidados fue en gran medida consecuencia de la entrega de los profesionales de salud mental que los pusieron en marcha, sin embargo el mantenimiento de estos programas no puede basarse en la entrega personal. Se necesita una profesionalización que garantice el mantenimiento de los programas más allá del voluntarismo de sus primeros impulsores. Por otra parte, el trabajo de los profesionales en salud mental comunitaria y, por tanto, en continuidad de cuidados requiere una gran flexibilidad, trabajo en medios diversos y alejarse de formas de atención más tradicionales basadas en el paternalismo y la seguridad hospitalaria. En un momento en el que la mirada vuelve al hospital y lo biomédico toma ventaja a lo biopsicosocial, el trabajo en continuidad de cuidados puede correr el peligro de ser considerado de segunda categoría.

\section{Referencias}

Consejería de Sanidad. Comunidad de Madrid. (2002). Plan de Salud Mental de la Comunidad de Madrid 2003-2008. Madrid: Consejería de Sanidad.

Consejería de Sanidad. Comunidad de Madrid (2010). Plan de Salud Mental de la Comunidad de Madrid 2010-2014. Madrid: Consejería de Sanidad.

Consejería de Servicios Sociales. Comunidad de Madrid. (2003). Plan de Atención Social a personas con enfermedad mental grave y crónica 2003-2007. Madrid: Consejería de Servicios Sociales.

Gehrs, M., \& Goering, P. (1994). The relationship between the working alliance and rehabilitation outcomes of schizophrenia. Psychosocial Rehabilitation Journal, 18, 43-54.

Klinkenberg, W. D., Calsyn, R. J., \& Morse, G. A. (1998). The helping alliance in case management for homeless persons with severe mental illness. Community Mental Health Journal, 34, 569-578.

McGrew, J. H., Wilson, R., \& Bond, G. R. (1996). Client perspectives on helpful ingredients of assertive community treatment. Psychiatric Rehabilitation Journal, 19(3), 13-21.

Neale, M. S., \& Rosenheck, R. A. (1995). Therapeutic alliance and outcome in a VA intensive case management program. Psychiatric Services, 46, 719-721.

Sobrino, T., Moreno, A., \& Desviat, M. (2007). Programa de Continuidad de Cuidados y Apoyo Comunitario de los Servicios de Salud Mental del Área 9. Leganés. Documento de trabajo no publicado.

Solomon, P., \& Draine, J. (1994). Satisfaction with mental health treatment in a randomized trial of consumer case management. Journal of Nervous and Mental Disease, 182, 179-184.

Thornicroft, G., Alem, A., Antunes dos Santos, R., Barley, E., Drake R. E., Gregorio, G., ... Wondimagegn, D. (2010). WPA guidance on steps, obstacles and mistakes to avoid in the implementation of community mental health care. World Psychiatry, 9(2),67-77. 